\title{
TRAVELING WAVE SOLUTIONS OF A MATHEMATICAL MODEL FOR TUMOR ENCAPSULATION*
}

\author{
JONATHAN A. SHERRATT ${ }^{\dagger}$
}

\begin{abstract}
The formation of a capsule of dense, fibrous extracellular matrix around a solid tumor is a key prognostic indicator in a wide range of cancers. However, the cellular mechanisms underlying capsule formation remain unclear. One hypothesized mechanism is the "expansive growth hypothesis," which suggests that a capsule may form by the rearrangement of existing extracellular matrix, without new matrix production. A mathematical model was recently proposed to study the implications of this hypothesis [Perumpanani, Sherratt, and Norbury, Nonlinearity, 10 (1997), pp. 1599-1614]. The model consists of conservation equations for tumor cells and extracellular matrix and exhibits traveling wave solutions in which a pulse of extracellular matrix, corresponding to a capsule, moves in parallel with the advancing front of the tumor. In this paper the author presents a detailed study of traveling wave behavior in the model, deriving conditions for the existence of traveling waves and their key properties. Numerical methods for solving the model equations are presented, and numerical simulations suggest that the traveling waves are stable and are the biologically relevant solution form for the model. The analytical results are extended to an improved model, which includes a saturation in the extent of matrix rearrangement per cell. Finally, the author discusses the biological implications of the model results.
\end{abstract}

Key words. traveling waves, cancer, mathematical model

AMS subject classifications. 92C50, 92C15, 35M10, 34C05

PII. S0036139998345355

1. Introduction. Solid tumors typically undergo an initial period of avascular growth, after which they become quiescent for a long period. The dormant state is ended by invasion into surrounding tissue and the onset of angiogenesis, the first steps in the metastatic cascade. A very significant feature of the quiescent phase is the presence, in some cases, of capsules of dense, fibrous extracellular matrix around the tumor (illustrated in Figure 1). For example, in a detailed study of hepatocellular carcinoma, $\mathrm{Ng}$ et al. [12] found that about half (72/154) of tumors were encapsulated. The presence of a capsule is a key prognostic indicator, and this is thought to be due to the capsule acting simply as a physical barrier to invasion and angiogenesis. Recent survival data comparisons for encapsulated and nonencapsulated tumors are available for a variety of cancers [5], [8], [10], [12]; in particular, in Lai et al.'s [8] data for large hepatocellular carcinoma, tumor encapsulation was identified as the only important favorable prognostic feature relating to disease-free survival.

Despite this major clinical significance of tumor encapsulation, the mechanisms responsible for capsule formation remain unclear. In particular, it is debated whether remodeling of existing extracellular matrix alone is responsible for the capsule (the "expansive growth hypothesis"), or whether de novo cellular secretion of collagen plays a key role (the "foreign body hypothesis"). Evidence on either side of this controversy comes primarily from pathology data. For example, Wakasa et al. [17] found that hepatocellular carcinomas were encapsulated more frequently when their diameter was

\footnotetext{
*Received by the editors October 2, 1998; accepted for publication (in revised form) February 11, 1999; published electronically December 28, 1999. This work was supported in part by grant GR/L41967 from the EPSRC (Applied Nonlinear Mathematics Initiative) and in part by a Scheme 3 grant from the London Mathematical Society.

http://www.siam.org/journals/siap/60-2/34535.html

${ }^{\dagger}$ Department of Mathematics, Heriot-Watt University, Edinburgh EH14 4AS, UK (jas@ma. hw.ac.uk).
} 


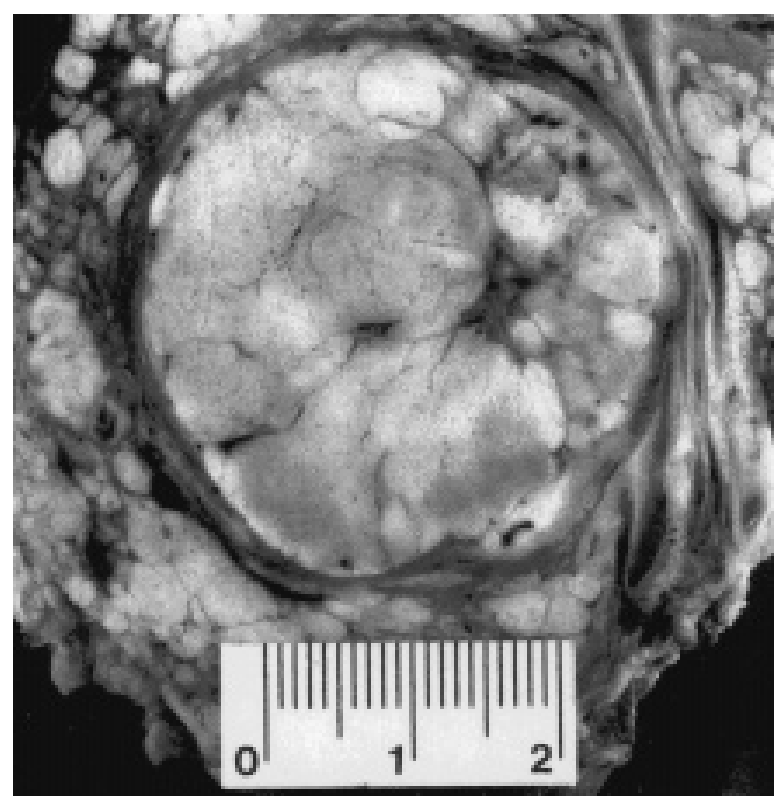

FIG. 1. The appearance of an encapsulated hepatocellular carcinoma, which has been removed surgically from a cirrhotic liver. The tumor is surrounded by a dense fibrous capsule, several millimeters thick (scale in centimeters). This figure is reproduced from $\mathrm{Ng}$ et al., Cancer, 70 (1992), pp. 45-49. Copyright (C)(1992) American Cancer Society. Reprinted by permission of Wiley-Liss, Inc., a subsidiary of John Wiley 83 Sons, Inc.

greater than $2 \mathrm{~cm}$, which they took as evidence that the capsule forms by compression of adjacent extracellular matrix. However, Ng et al.'s [12] data showed no correlation between tumor size and either capsule incidence or thickness. These contrasting results are hard to evaluate because of the difficulty of performing experimental tests. One of the few such tests is in the work of Vaage and Harlos [16], who showed that production of collagen by macrophages is responsible for the encapsulation of tumor implants in mice; however, this may be different from the mechanisms involved for naturally arising tumors.

In a situation such as this, where experimental investigation is very difficult, mathematical modeling provides a convenient way of testing hypotheses. This approach was first applied to capsule formation in the recent paper of Perumpanani, Sherratt, and Norbury [14], who developed a model to study the "expansive growth hypothesis." Their model consists of conservation equations for the densities of tumor cells and extracellular matrix, denoted $u(x, t)$ and $c(x, t)$, respectively, where $t$ and $x$ denote time and space in a one-dimensional spatial domain:

$$
\begin{aligned}
\frac{\partial u}{\partial t} & =f(u)+\frac{\partial}{\partial x}\left[h(c) \frac{\partial u}{\partial x}\right], \\
\frac{\partial c}{\partial t} & =k \frac{\partial}{\partial x}\left[\operatorname{ch}(c) \frac{\partial u}{\partial x}\right] .
\end{aligned}
$$

Here the term $f(u)$ represents cell division and death; $f(0)$ must clearly be zero, and for simplicity I will assume throughout that the cell density is rescaled so that $u=1$ is the equilibrium level within the tumor, implying $f(1)=0$; Perumpanani, Sherratt, 
and Norbury take $f(u)=u(1-u)$. Random cell movement is assumed, and kinetics of extracellular matrix are neglected, in keeping with the expansive growth hypothesis, so that the extracellular matrix density only changes because of convection with the cells. This convection does not imply large-scale movement of intact matrix by a cell; rather it is the net result of local matrix movement and remodeling during cell movement. This will increase with the local matrix density and is represented in the model as $k \cdot c$. In practice, this term will saturate at high matrix densities, representing the limitation on matrix reorganization per cell. Perumpanani, Sherratt, and Norbury neglected it for simplicity; I will do likewise initially but consider its inclusion later in the paper.

The function $h(c)$ is decreasing and is included in the model to represent the reduction in cell motility at high matrix densities. Although Perumpanani, Sherratt, and Norbury [14] included this function when developing the model, their analysis was restricted to the case of $h($.$) being constant, which greatly simplifies (1) by decoupling$ the two equations. In this paper, I investigate the case of nonconstant $h($.$) , studying$ the existence and form of traveling wave solutions. I begin by summarizing the results of Perumpanani, Sherratt, and Norbury [14] in section 2. Behavior in the case of nonconstant $h($.$) depends on whether h(\infty)$ is zero or nonzero, and I consider these cases in sections 3 and 4 , respectively. In section 5 , I discuss numerical methods for solving (1) and present numerical solutions to illustrate my analytical results. Finally, in section 6 I consider extending the model to include saturation in the extent of matrix reorganization per cell.

2. Solutions for constant $\boldsymbol{h}($.$) . When h($.$) is constant, (1a) becomes indepen-$ dent of $c$, and for the case $f(u)=u(1-u)$ considered in [14], it has the form of the much-studied Fisher equation (see, for example, [7], [9], [1], [15]). In the context of tumor growth, one is concerned with the evolution of (1) from initial data corresponding to a localized population of tumor cells, and a uniform (nonzero) density of matrix, which I take to be $c \equiv 1$. For simplicity, Perumpanani, Sherratt, and Norbury take this tumor cell population to be at one boundary of a semi-infinite domain, with a noflux boundary condition. When $u$ satisfies the Fisher equation, such initial conditions for $u$ evolve to traveling wave fronts, moving out from the boundary with constant shape and speed (Figure 2(a)). The details of this evolution are very well known; for example, analytical expressions for rates of convergence have been calculated [9]. The wave front itself is of course a function of a traveling wave coordinate $z=x-a t$, where $a$ is the wave speed ( $a=2 \sqrt{h}$ when $h$ is constant), although there is no closed form expression for this wave.

In order to study the long term behavior of (1) with appropriate initial conditions, Perumpanani, Sherratt, and Norbury substituted the traveling wave form for $u, u_{t w}(z)$ say, into (1b), giving a first-order partial differential equation for $c$. This equation is most conveniently written using independent variables $z$ and $t$ and can be solved using the method of characteristics. This shows that the qualitative behavior depends crucially on whether $k$ is above or below the critical value $a / \max _{-\infty<z<\infty}\left\{-h u_{t w}^{\prime}(z)\right\}$. For $k$ below this value, $c$ also evolves to a permanent form traveling wave solution (Figure 2(b)); the wave form is in fact given by the simple expression $c_{t w}(z)=a /\left[a+k h u_{t w}^{\prime}(z)\right]$. However, for $c$ above the critical value, the solution for $c$ again has a wave form but in this case not of constant shape. Rather, the height of the peak in this wave front grows, without limit, as time increases (Figure 2(c)); the analytical solution shows that the rate of growth is exponential. Such growth is entirely consistent with the assumption of no matrix production: the peak 


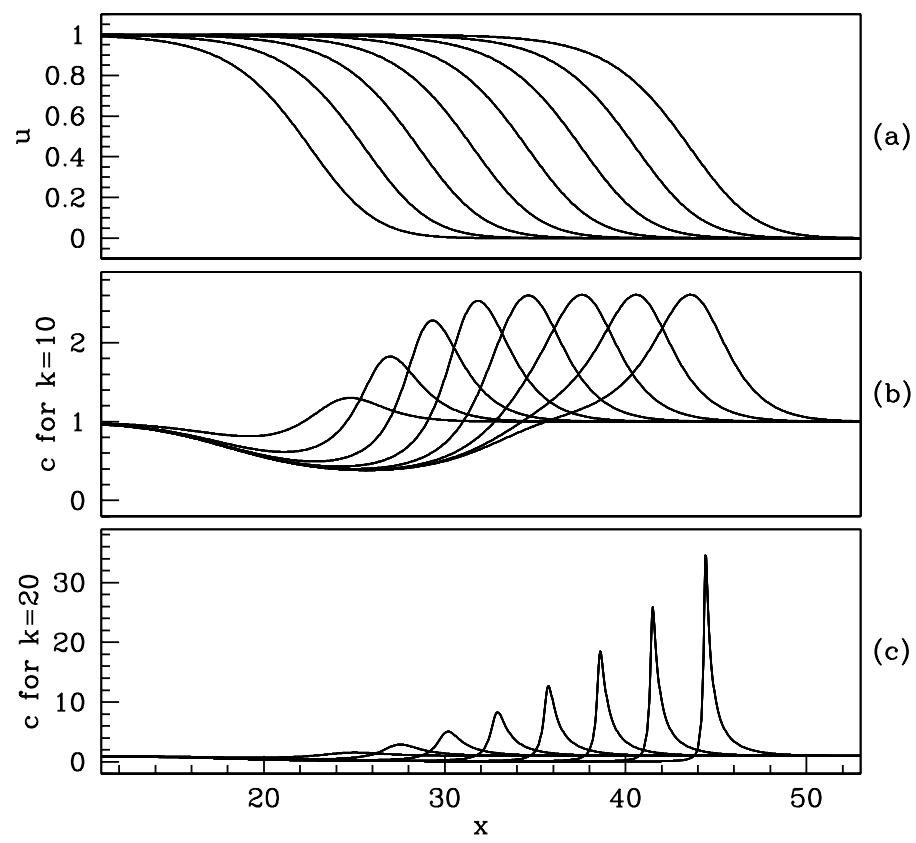

FIG. 2. Illustration of the solution types of (1) when $h(c) \equiv 1$. The cell density $u$ satisfies Fisher's equation and evolves rapidly to a traveling wave front for biologically appropriate initial conditions (a). For $k$ below a critical value (which is approximately 16.1), the extracellular matrix density $c$ also evolves to a traveling wave form (b), while for $k$ above this critical value, the peak in c continues to increase in height without limit (c). The solutions plotted are calculated from the exact solutions derived by Perumpanani, Sherrat, and Norbury in [14] by assuming a permanent form traveling wave solution for $u(x, t), u_{t w}(z)$, say, where $z=x-a t ; a$ is the wave speed, which is determined by the $u$ equation, and is 2 for biologically realistic initial conditions. Substitution of this solution for $u$ into (1b) gives a first-order partial differential for $c(x, t)$, which can be solved using the method of characteristics. The solution illustrated is for the case $c(x, 0) \equiv 1$, in which case the solution derived in [14] is $c(x, t)=c_{t w}(\theta) / c_{t w}(z)$, where $c_{t w}(z) \equiv a /\left[a+k u_{t w}^{\prime}(z)\right]$. The variable $\theta \equiv G^{-1}[G(z)-t]$, where $G(z) \equiv-\int c_{t w}(z) d z$, with $G^{-1}($.$) a local inverse. In order to plot this$ solution, the traveling wave ODE for $u$ was integrated numerically, enabling numerical integration of $G($.$) and hence calculation of c_{t w}, \theta$, and thus $c$. The solution is plotted as a function of $x$ at equally spaced times $(t=1.0,2.5,4.0,5.5,7.0,8.5,10.0,11.5)$.

becomes thinner as it grows, so that the total density of matrix within it is constant.

The assumption that $h($.$) is constant is mathematically very convenient, but$ biologically it is unrealistic, since in practice rates of cell movement are significantly affected by local extracellular matrix [2]. In the next two sections, I consider traveling wave behavior in the more general case, studying separately the cases of $h(\infty)$ zero and nonzero. My results depend on the assumptions that $h($.$) and f($.$) are continuously$ differentiable and satisfy the following conditions:

- $f(0)=f(1)=0$ with $f(u)>0$ for $u \in(0,1)$;

- $f^{\prime}(0)>0$ and $f(u)<f^{\prime}(0) u$ for $u \in(0,1]$;

- $f(u)$ has only one turning point on $(0,1)$ at $u=u_{m}$, say;

- $h(c) \geq 0$ and $h^{\prime}(c) \leq 0$ for $c \geq 0$ with $h(1)>0$.

Conditions (2a) and (2b) are standard assumptions for cell kinetic terms, made orig- 
inally by Kolmogoroff, Petrovskii, and Piscounoff [7] in their work on scalar reactiondiffusion equations. The additional constraint (2c) facilitates the calculations in section 2 , and is not at all restrictive from the viewpoint of applications.

3. Traveling waves when $\boldsymbol{h}(\infty)=\mathbf{0}$. Traveling wave solutions of (1) have the form $u(x, t)=U(x-a t), c(x, t)=C(x-a t)$, where $a$ is the wave speed, and thus satisfy the ordinary differential equations

$$
\begin{aligned}
f(U)+a U^{\prime}+\left[U^{\prime} h(C)\right]^{\prime} & =0, \\
a C^{\prime}+k\left[C U^{\prime} h(C)\right]^{\prime} & =0 .
\end{aligned}
$$

Here prime denotes $d / d z$, with $z=x-a t$ a traveling wave coordinate; the wave speed $a$ enters the equations as a parameter, and I take $a>0$ without loss of generality. In the context of tumor growth, one is concerned with solutions of these equations satisfying $U \rightarrow 0, C \rightarrow 1$ as $z \rightarrow \infty$, and $U \rightarrow 1, C \rightarrow 1$ as $z \rightarrow-\infty$ with $U \geq 0$ for all $z$; the wave corresponds to the advancing front of the tumor edge. It is a solution of this type that is illustrated in Figure 2(a). In this section I will prove that when $h(\infty)=0$, and with $f($.$) and h($.$) satisfying conditions (2), equations (3) have a$ solution of this type if and only if $a \geq 2\left[f^{\prime}(0) h(1)\right]^{1 / 2}$; the case $h(\infty)>0$ is considered in the next section.

Equation (3b) can be integrated immediately, and since $U^{\prime} \rightarrow 0, C \rightarrow 1$ as $z \rightarrow \pm \infty$, this gives

$$
U^{\prime} h(C)=\frac{a}{k}\left(\frac{1}{C}-1\right) .
$$

To proceed, I must rewrite this to give $h(C)$ as a function of $U^{\prime}$, which can then be substituted into (3a). Since $1 / h(C)$ and $(1-1 / C)$ are, respectively, increasing and strictly increasing functions of $C,(4)$ implies that $U^{\prime}$ is a strictly decreasing function of $C$. Moreover, I have $\left.U^{\prime}\right|_{C=0}=\infty,\left.U^{\prime}\right|_{C=1}=0$, and $\left.U^{\prime}\right|_{C=\infty}=-\infty$; the last of these limits depends on $h(\infty)$ being zero (Figure 3(a), (b)). Inverting this implies that $C$ is a strictly decreasing function of $U^{\prime}$ (Figure $3(\mathrm{c})$ ) and hence $(1 / C-1) \cdot a / k \equiv U^{\prime} h(C)$ is a strictly increasing function of $U^{\prime}$, which I denote by $\Phi\left(U^{\prime}\right)$ (Figure $3(\mathrm{~d})$ ). The precise form of $\Phi($.$) depends on h($.$) of course, but in all cases \Phi(-\infty)=-a / k$, $\Phi(0)=0$, and $\Phi(\infty)=\infty$.

Substituting the expression $U^{\prime} h(C)=\Phi\left(U^{\prime}\right)$ into (3a) gives

$$
f(U)+a U^{\prime}+\Phi^{\prime}\left(U^{\prime}\right) U^{\prime \prime}=0 .
$$

The form of $\Phi^{\prime}($.$) is key to subsequent calculations. Differentiating (4) with respect$ to $U^{\prime}$ gives

$$
\begin{aligned}
h(C)+U^{\prime} h^{\prime}(C) \frac{\partial C}{\partial U^{\prime}}=\frac{-a}{k} \frac{\partial C}{\partial U^{\prime}} \frac{1}{C^{2}} \\
\Rightarrow \frac{\partial C}{\partial U^{\prime}}=\frac{-k C^{2} h(C)}{k C^{2} U^{\prime} h^{\prime}(C)+a} \\
=\frac{-k C^{2} h(C)^{2}}{a h(C)+a C(1-C) h^{\prime}(C)}
\end{aligned}
$$



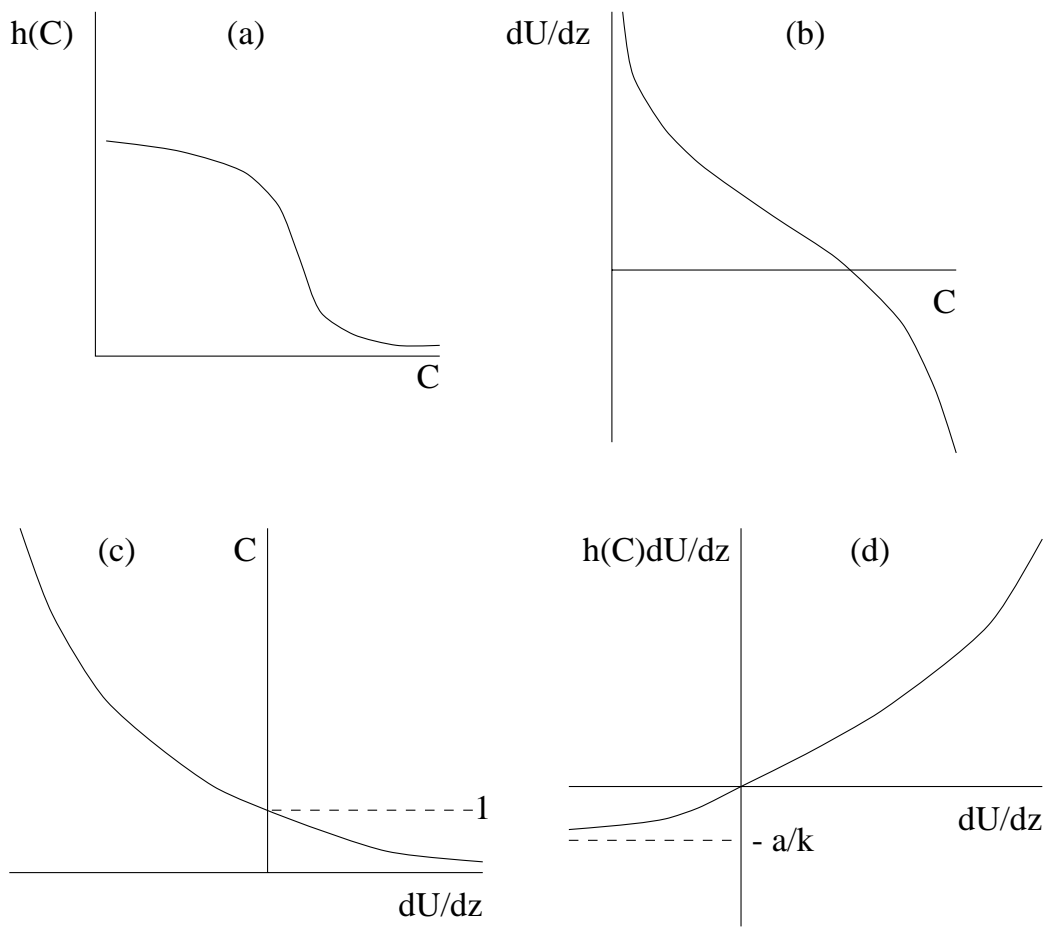

Fig. 3. Schematic illustration of the derivation of the form of $U^{\prime} h(C)$ as a function of $U^{\prime}$, from $(4)$, when $h(\infty)=0$. (a) The form of $h(C)$ as a function of $C$. (b) The form of $U^{\prime} h(C) \equiv$ $(1 / C-1) \cdot a / k$ as a function of $C$. (c) The form of $C$ as a function of $U^{\prime}$. (d) The form of $\Phi\left(U^{\prime}\right) \equiv U^{\prime} h(C) \equiv(1 / C-1) \cdot a / k$ as a function of $U^{\prime}$.

using (4). Therefore

$$
\begin{aligned}
\Phi^{\prime}\left(U^{\prime}\right) & \equiv \frac{\partial}{\partial U^{\prime}}\left[\frac{a}{k}\left(\frac{1}{C}-1\right)\right] \\
& =\frac{-a}{k C^{2}}\left\{\frac{\partial C}{\partial U^{\prime}}\right\} \\
& =\left\{\frac{1}{h(C)}+\frac{C(1-C) h^{\prime}(C)}{h(C)^{2}}\right\}^{-1},
\end{aligned}
$$

where $C$ is determined as a function of $U^{\prime}$ from (4) and has the qualitative form illustrated in Figure 3(c). In particular, note that $\Phi^{\prime}(0)=h(1)$.

Armed with this formula for $\Phi^{\prime}($.$) , I can now complete the proof of the existence of$ a positive traveling wave solution by studying (5) in a phase plane. This next section of the proof is an adaptation of methods developed in [7] for studying traveling wave solutions of Fisher's equation. I rewrite (5) as

$$
U^{\prime}=W / k, \quad W^{\prime}=-\gamma(W)[k f(U)+a W],
$$

where $\gamma(W)=1 / \Phi^{\prime}(W / k)$. This notation is chosen to make the dependence on the parameter $k$ clearer - this will be important in the next section. Note that when written as a function of $W, \gamma($.$) has no explicit dependence on k$.

It is straightforward to show that for functions $f($.$) satisfying (2),(7)$ has two equilibria: $(1,0)$ which is a saddle point, and $(0,0)$, which is a stable node if $a \geq$ 
$2\left[f^{\prime}(0) / \gamma(0)\right]^{1 / 2}=2\left[f^{\prime}(0) h(1)\right]^{1 / 2}$, and a stable focus otherwise. Thus there is a unique trajectory $\mathcal{T}$ leaving $(1,0)$ and entering the lower half-plane. The traveling wave solutions I am considering correspond to connections between the two equilibria and thus to the trajectory $\mathcal{T}$. Hence there is at most one wave for any given $a$, with the wave existing if and only if $\mathcal{T}$ terminates at $(0,0)$; this cannot occur with $U \geq 0$ when $(0,0)$ is a spiral. When $a \geq 2\left[f^{\prime}(0) h(1)\right]^{1 / 2}$, there are three possible ways in which $\mathcal{T}$ can leave the lower right quadrant $(U>0, W<0)$. First, $\mathcal{T}$ can pass through the positive $U$-axis. At the point of this crossing, $U>0, W=0$, and $W^{\prime}>0$, which contradicts (7). Second, $\mathcal{T}$ can leave the lower right quadrant through the negative $W$-axis. In this case, $\mathcal{T}$ must first cross the line $W=\lambda k U$, where $\lambda$ is one of the eigenvalues at $(0,0)$, which are real and negative for $a$ above the critical value. At the point of this crossing

$$
\begin{aligned}
d W / d U & =-a k \gamma(W)-k^{2} f(U) \gamma(W) / W \\
& <-a k \gamma(W)-k^{2} U f^{\prime}(0) \gamma(W) / W \quad \text { using (2) } \\
& =-a k \gamma(W)-k \gamma(W) f^{\prime}(0) / \lambda \quad \text { since } W=\lambda k U \\
& =\lambda k \gamma(W) / \gamma(0)
\end{aligned}
$$

using the eigenvalue equation $\lambda^{2}+a \gamma(0) \lambda+\gamma(0) f^{\prime}(0)=0$. The relationship between $C$ and $U^{\prime}$ implies that $C>1$ when $W<0$, and since $h^{\prime}(C) \leq 0$, the formula (6) shows that when $C>1$,

$$
\gamma(W) \geq 1 / h(C) \geq 1 / h(1)=\gamma(0) .
$$

Therefore when $\mathcal{T}$ crosses the line $W=\lambda k U, d W / d U<\lambda k$ (recall that $\lambda<0$ ), which is a contradiction.

Thus only the third possibility remains, namely, that whenever $a \geq 2\left[f^{\prime}(0) h(1)\right]^{1 / 2}$, $\mathcal{T}$ leaves the region $U>0, W<0$ by terminating at $(0,0)$, corresponding to a traveling wave solution for $U(z)$, which is monotonically decreasing. The corresponding solution for $C(z)$ is determined by its relationship with $U^{\prime}(z)$, which is illustrated in Figure $3(\mathrm{c}) ; C(z)$ is a pulse wave form.

4. Traveling waves when $\boldsymbol{h}(\infty)>\mathbf{0}$. When $h(\infty)>0$, many of the arguments of the previous section continue to hold. In particular, traveling wave solutions still correspond to heteroclinic connections in the system (7), and the proof that such connections exist whenever $a \geq 2\left[f^{\prime}(0) h(1)\right]^{1 / 2}$ still holds. The key difference lies in the relationship between $U^{\prime}$ and $C$, which no longer has the qualitative form illustrated in Figure 3(c). In this case, $1 / h(C)$ approaches a finite asymptote as $C \rightarrow \infty$, and thus $U^{\prime}$ is a strictly decreasing function of $C$, approaching $-a /[k h(\infty)]$ as $C \rightarrow \infty$ (Figure 4(a), (b)). Inverting this implies that $C$ is a strictly decreasing function of $U^{\prime}$ but is defined only for $U^{\prime}>-a /[k h(\infty)]$ (Figure 4(c)); the corresponding form of $\Phi\left(U^{\prime}\right)$ is illustrated in Figure 4(d). The formula (6), giving the form of $\gamma($.$) , continues to hold,$ and $\gamma$ may either approach a finite (positive) value or $+\infty$ as $U^{\prime} \rightarrow-a /[k h(\infty)]^{+}$, depending on the form of $h(C)$ as $C \rightarrow \infty$.

The constraint on $U^{\prime}$ for $C$ to be defined means that when $h(\infty)>0$, the trajectory $\mathcal{T}$ in the $U-W$ phase plane only gives a solution of (3) when it lies entirely above the line $W=-a / h(\infty)$. In this section, I will show that this occurs if and only if $k$ is less than a critical value $k_{c r i t}$, which depends on the details of $h($.$) and f($.$) .$ To demonstrate this, I will prove the following proposition:

(i) $\mathcal{T}$ has a unique minimum in the $U-W$ plane, at the point $\left(U_{\min }, W_{\min }\right)$, say. 

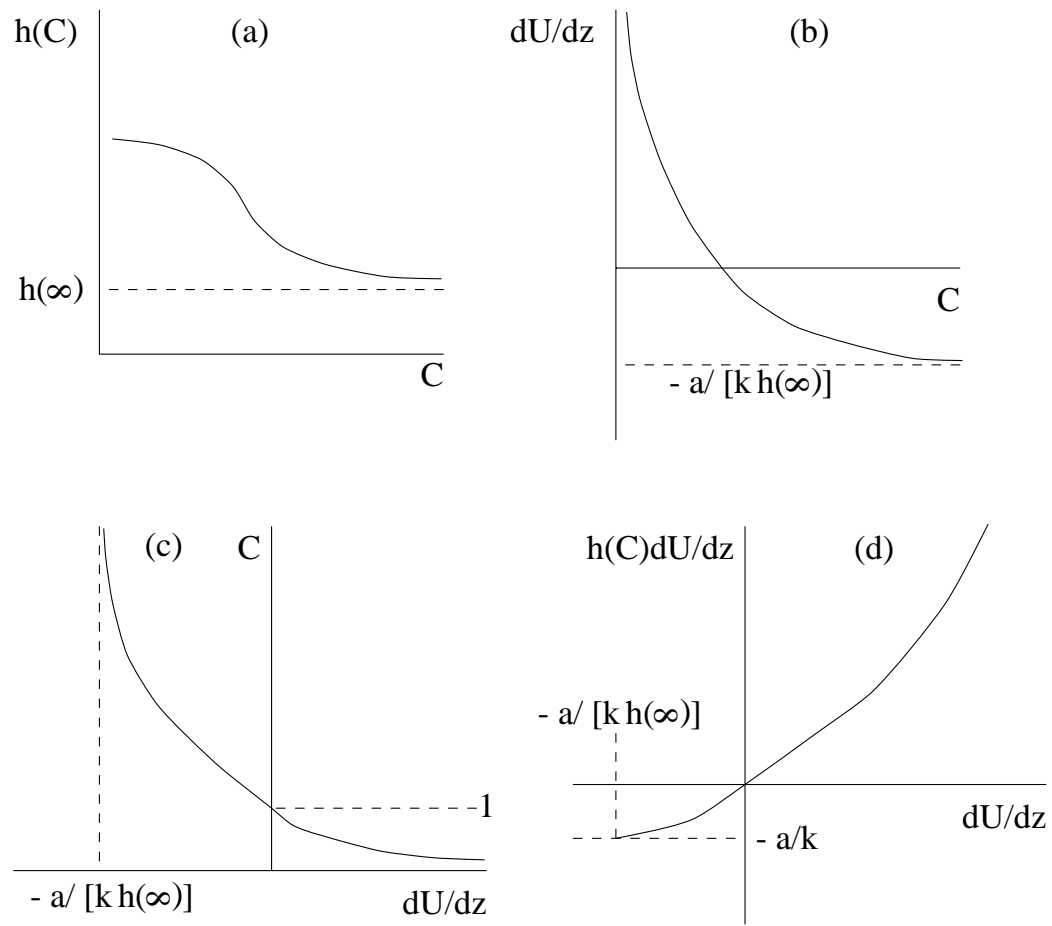

FIG. 4. Schematic illustration of the derivation of the form of $U^{\prime} h(C)$ as a function of $U^{\prime}$, from (4), when $h(\infty)>0$. (a) The form of $h(C)$ as a function of $C$. (b) The form of $U^{\prime} h(C) \equiv$ $(1 / C-1) \cdot a / k$ as a function of $C$. (c) The form of $C$ as a function of $U^{\prime}$. (d) The form of $\Phi\left(U^{\prime}\right) \equiv U^{\prime} h(C) \equiv(1 / C-1) \cdot a / k$ as a function of $U^{\prime}$.

(ii) $W_{\min }(<0)$ is a strictly decreasing function of $k$.

(iii) $W_{\text {min }} \rightarrow 0$ as $k \rightarrow 0$, and $W_{\min } \rightarrow-\infty$ as $k \rightarrow \infty$.

Here it is implicitly assumed that $a \geq 2\left[f^{\prime}(0) h(1)\right]^{1 / 2}$, so that $\mathcal{T}$ exists. Note that a simple consequence of this proposition is that the traveling pulse solution for $C$ has a unique maximum, whose height increases strictly with $k$, tending to $\infty$ as $k \rightarrow k_{\text {crit }}^{-}$.

Proof of (i). I begin by observing that $d W / d U>0$ if and only if $W$ lies above the curve $W=-k f(U) / a$, which I refer to as $\mathcal{C}_{1}$. Now the eigenvector along which $\mathcal{T}$ leaves $(1,0)$ has a slope equal to the product of $k$ and the corresponding eigenvalue, which is equal to

$$
\begin{aligned}
\frac{k}{a}\left[-\frac{1}{2} a^{2} \gamma(0)+\sqrt{\frac{1}{4} a^{4} \gamma(0)^{2}-a^{2} \gamma(0) f^{\prime}(1)}\right] & <\frac{k}{a}\left[-\frac{1}{2} a^{2} \gamma(0)+\sqrt{\left[\frac{1}{2} a^{2} \gamma(0)-f^{\prime}(1)\right]^{2}}\right] \\
& =-k f^{\prime}(1) / a,
\end{aligned}
$$

which is the slope of $\mathcal{C}_{1}$ at $(1,0)$. Thus $\mathcal{T}$ lies above $\mathcal{C}_{1}$ when $U$ is close to 1 . $\mathcal{T}$ will have a turning point when it crosses $\mathcal{C}_{1}$, and at such a point, $\mathcal{T}$ will be horizontal in the $U-W$ plane. Hence the first turning point must occur when the slope of $\mathcal{C}_{1}$ is negative, that is, when $U<u_{m}$; recall that I am assuming that $f(U)$ has a unique local maximum at $U=u_{m}$. $\mathcal{T}$ must have at least one turning point of course, and I denote the first turning point by $\left(U_{\min }, W_{\text {min }}\right)$. A subsequent turning point would again require $\mathcal{T}$ to cross $\mathcal{C}_{1}$ horizontally. This is impossible since $U_{\min }<u_{m}$, so that the slope of $\mathcal{C}_{1}$ is positive for $U<U_{\text {min }}$; recall that $U$ decreases monotonically along 
$\mathcal{T}$. Hence there is a unique turning point, as required. Proof of (ii). Equation (7) implies that

$$
\frac{\partial}{\partial k}\left[\frac{d W}{d U}\right]=-\gamma(W)[a+2 k f(U) / W]
$$

which is positive if and only if $W$ lies between the $U$-axis and the curve $W=$ $-2 k f(U) / a$, which I refer to as $\mathcal{C}_{2}$. Note that the value of $W$ on $\mathcal{C}_{2}$ is exactly twice that on $\mathcal{C}_{1}$ for each $U$. Now consider two values of $k, k^{*}$, and $k^{* *}$, say, satisfying $k^{*}<k^{* *}<2 k^{*}$; I use the superscripts ${ }^{*}$ and ${ }^{* *}$ to denote the corresponding curves and trajectories. Then the minimum of $\mathcal{T}^{* *}$ lies on the curve $\mathcal{C}_{1}^{* *}$, which lies between the $U$-axis and the curve $\mathcal{C}_{2}^{*}$, since $k^{* *}<2 k^{*}$. Thus the portions of the trajectories $\mathcal{T}^{*}$ and $\mathcal{T}^{* *}$ between $(1,0)$ and their respective minima both lie in a region of the phase plane within which $\frac{\partial}{\partial k}(d W / d U)>0$ for $k \in\left[k^{*}, k^{* *}\right]$. This implies that this portion of $T^{*}$ lies strictly between the $U$-axis and the corresponding portion of $T^{* *}$, so that $W_{\min }^{* *}<W_{\min }^{*}$ as required. Since $k^{*}$ and $k^{* *}$ are arbitrary within the specified restrictions, this implies the required monotonicity.

Proof of (iii). This follows immediately from the fact that $d W / d U \rightarrow \infty$ as $k \rightarrow \infty$, and $d W / d U \rightarrow 0$ as $k \rightarrow 0$ for all $U \in(0,1)$ and $W>0$.

When $k \geq k_{\min }$, no traveling wave solution exists. I have been unable to make any analytical progress in studying equations (1) in this case: in particular, the approach used by Perumpanani, Sherratt, and Norbury [14], and described in section 2, cannot be applied when $h($.$) is nonconstant. Therefore I rely on numerical solutions, which I$ will discuss in the next section.

5. Numerical solution of the model equations. The system (1) is of mixed type, because of the coupling of hyperbolic and parabolic terms, and this makes numerical solution difficult when $h($.$) is nonconstant. From a practical viewpoint, the$ problem requires particular care because I have found that a number of inappropriate schemes produces results which, although incorrect, are superficially plausible. The key to a successful numerical scheme is the spatial discretization, which must involve upwinding for the convective term in (1b) and must reflect the conservation of $c$ exactly. I have used a fixed spatial grid, and denoting the values of $c$ at the $N$ grid points by $c_{i}(i=1, \ldots, N)$, my numerical scheme involves the following system of coupled ordinary differential equations:

$$
\begin{gathered}
\frac{\partial u_{i}}{\partial t}=\frac{1}{\delta}\left[h\left(c_{i+1 / 2}\right) u_{x, i+1 / 2}-h\left(c_{i-1 / 2}\right) u_{x, i-1 / 2}\right]+f\left(u_{i}\right), \\
\frac{\partial c_{i}}{\partial t}=\frac{k}{\delta}\left\{\left[\Gamma_{i+1}-\Gamma_{i}\right] \cdot \mathcal{I}\left[H^{\prime}\left(c_{i+1 / 2}\right)<0\right]\right. \\
\left.\quad+\left[\Gamma_{i}-\Gamma_{i-1}\right] \cdot \mathcal{I}\left[H^{\prime}\left(c_{i-1 / 2}\right)>0\right]\right\}
\end{gathered}
$$

Here $\delta$ is the grid spacing, $H(c) \equiv c \cdot h(c)$, and $\Gamma_{i} \equiv H\left(c_{i}\right) u_{x, i}$; derivatives of $H($. can be evaluated analytically for a given form of $h($.$) . \mathcal{I}$ is the indicator function, defined by $\mathcal{I}$ [.TRUE.] $=1, \mathcal{I}$ [.FALSE.] $=0$. The subscripts $i \pm 1 / 2$ are used in the standard way, to denote average values at the grid points on either side, and the subscript $x$ denotes a partial derivative, calculated using central differences. The form of upwinding used in (8b) is standard for scalar equations [11], and the details of this extension to a coupled system are crucial in order to preserve conservation of $c$. This requires particularly careful investigation when $H^{\prime}\left(c_{i \pm 1 / 2}\right)$ have different signs: 
To be specific, suppose that $H^{\prime}\left(c_{i-1 / 2}\right)$ is positive when $i=I, I-1, I-2, \ldots$, and negative when $i=I+1, I+2, \ldots$ Equation (8) then implies

$$
\begin{aligned}
& \frac{\partial}{\partial t}\left(\cdots+c_{I-2}+c_{I-1}+c_{I}+c_{I+1}+c_{I+2}+\cdots\right) \\
= & \cdots+\left(\Gamma_{I-2}-\Gamma_{I-3}\right)+\left(\Gamma_{I-1}-\Gamma_{I-2}\right)+\left(\Gamma_{I+1}-\Gamma_{I}+\Gamma_{I}-\Gamma_{I-1}\right) \\
+ & \left(\Gamma_{I+2}-\Gamma_{I+1}\right)+\left(\Gamma_{I+3}-\Gamma_{I+2}\right)+\cdots,
\end{aligned}
$$

so that $c$ is conserved. Similarly, conservation holds when $H^{\prime}$ changes sign in the opposite direction. Alternative discretizations of $(1 \mathrm{~b})$ are possible that give a smaller bandwidth for $u_{i}$ in the solution matrix, but these give loss of conservation of $c$ at isolated points, which leads to spurious solutions of (1).

I have solved the system (8) numerically using a standard stiff differential equation solver [3]. Recall that biologically appropriate initial conditions have $c \equiv 1$, and $u=0$ except for a local perturbation, corresponding to a small initial tumor, which I take to be at a boundary with no flux end conditions. Numerical solutions for such initial data reflect the analytical predictions of sections 2 and 3 . When $h(\infty)=0$, numerical solutions evolve to traveling wave forms in all cases I have tried (illustrated in Figure 5(a), (b)). This provides strong evidence for the stability of the traveling wave forms. Similarly, when $h(\infty)>0$ with $k$ small, a traveling wave front develops, while for $k$ above the critical value, the $c$ wave has a peak whose height increases continually with time, in a manner analogous to the behavior when $h($.$) is constant$ (Figure 5(c), (d)). Although I do not have a formula for $k_{\text {crit }}$, it is easy to calculate numerically, as the value of $k$ for which the $W_{\min }=-a / h(\infty)$, and Figure 6 illustrates the increase in height of the peak in the traveling wave solution $C(z)$ as $k$ increases toward $k_{\text {crit }}$ for one particular function $h($.$) .$

6. Model extension: Saturation in matrix remodeling rate. The model (1) assumes that the rate of matrix movement and convection per cell is directly proportional to the local matrix density. This is clearly a simplifying assumption, since in reality the term will saturate at high matrix densities. In this section I consider the effects of amending the model to include such saturation, by replacing the constant $k$ in (1b) by $k \cdot \theta(c)$, where $\theta(c)$ is a continuously differentiable decreasing function of $c$, with $\theta(1)=1$. This gives the amended model

$$
\begin{aligned}
& \frac{\partial u}{\partial t}=f(u)+\frac{\partial}{\partial x}\left[h(c) \frac{\partial u}{\partial x}\right], \\
& \frac{\partial c}{\partial t}=k \frac{\partial}{\partial x}\left[\operatorname{ch}(c) \theta(c) \frac{\partial u}{\partial x}\right] .
\end{aligned}
$$

The results proved in sections 3 and 4 can be easily extended to these equations also. The corresponding traveling wave equations imply

$$
\begin{aligned}
f(U)+a U^{\prime}+\left[U^{\prime} h(C)\right]^{\prime} & =0, \\
a C+k C h(C) \theta(C) U^{\prime} & =a,
\end{aligned}
$$

using notation as in section 3 . Thus

$$
f(U)+a U^{\prime}+\widetilde{\Phi}^{\prime}\left(U^{\prime}\right) U^{\prime \prime}=0,
$$

where $\widetilde{\Phi}(.) \equiv U^{\prime} h(C)$, with $C$ defined as a function of $U$ by (10b). Here, and in the remainder of this section, I adopt the notation of writing with a tilde the amended 

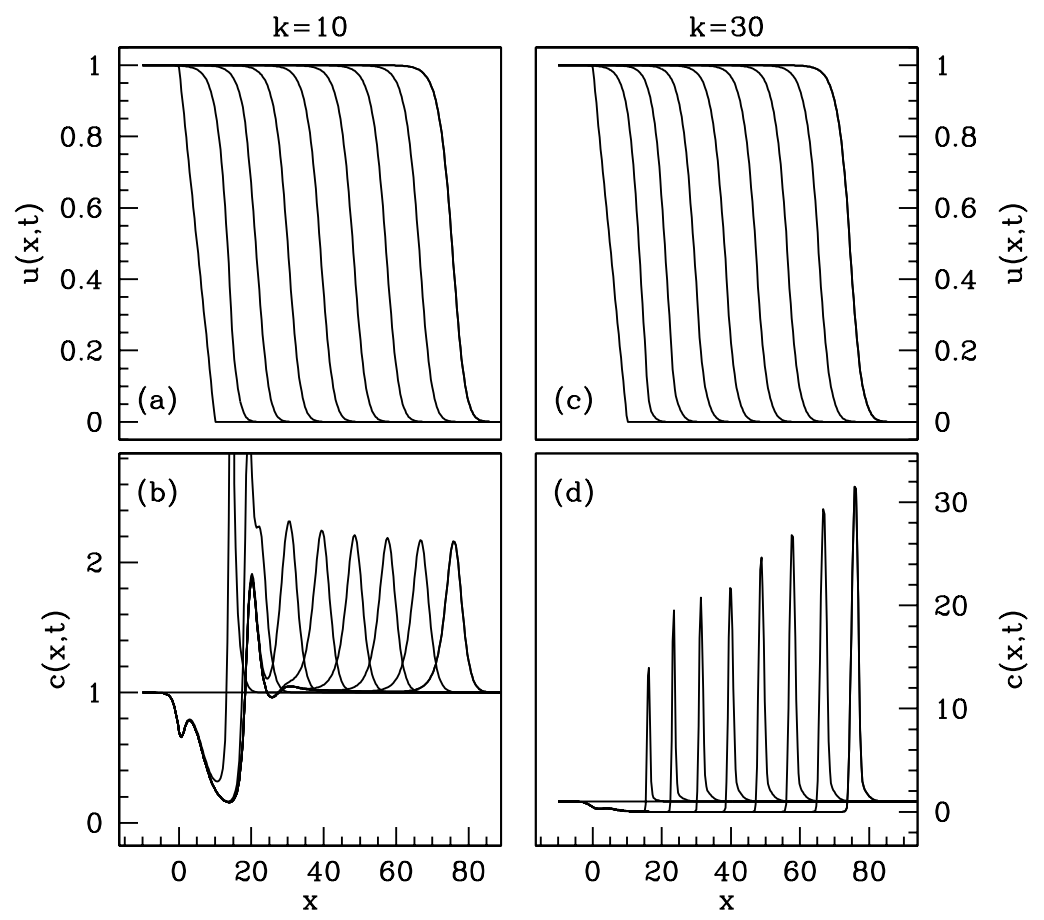

FIG. 5. Numerical solutions of (1) from initial conditions corresponding to a localized population of tumor cells. The solutions are plotted as a function of space $x$ at equally spaced time intervals (five dimensionless time units). (a), (b) Solution for a value of $k<k_{\text {crit }}$ : The solution evolves rapidly to the permanent form traveling wave, except for a localized variation in $c$ near the left-hand boundary. (c), (d) Solution for a value of $k>k_{\text {crit }}$ : The peak in $c$ increases in height without limit. The values illustrated are for $h(c)=[3-\tanh (c / 2-1)] / 4$, so that $h(\infty)=\frac{1}{2}$; for this form of $h(),. k_{c r i t} \approx 28$. The values of $k$ are 10 in (a), (b), and 30 in (c), (d). The equations were solved numerically using the scheme described in section 5.

forms of the various functions in sections 3 and 4 . Therefore

$$
\begin{aligned}
\widetilde{\Phi}^{\prime}\left(U^{\prime}\right) & \equiv \frac{\partial}{\partial U^{\prime}}\left[\frac{a}{k}\left(\frac{1}{C}-1\right) \frac{1}{\theta(C)}\right] \\
& =\frac{-a}{k C^{2} \theta(C)} \frac{\partial C}{\partial U^{\prime}}\left[1+C(1-C) \theta^{\prime}(C) / \theta(C)\right] \\
& =\frac{h(C)^{2} \theta(C)\left[1+\theta^{\prime}(C) C(1-C) / \theta(C)\right]}{h(C) \theta(C)+\left[h(C) \theta^{\prime}(C)+h^{\prime}(C) \theta(C)\right] C(1-C)} \\
& =\left\{\frac{1}{h(C)}+\frac{C(1-C) h^{\prime}(C)}{h(C)^{2}\left[1+C(1-C) \theta^{\prime}(C) / \theta(C)\right]}\right\}^{-1} .
\end{aligned}
$$

Here $\partial C / \partial U^{\prime}$ has been calculated using (10b) in a manner directly analogous to that used in section 3. Writing $W=k U^{\prime}$ then gives equations directly analogous to (7):

$$
U^{\prime}=W / k, \quad W^{\prime}=-\widetilde{\gamma}(W)[k f(U)+a W],
$$

where $\widetilde{\gamma}(W)=1 / \widetilde{\Phi}^{\prime}(W / k)$. 


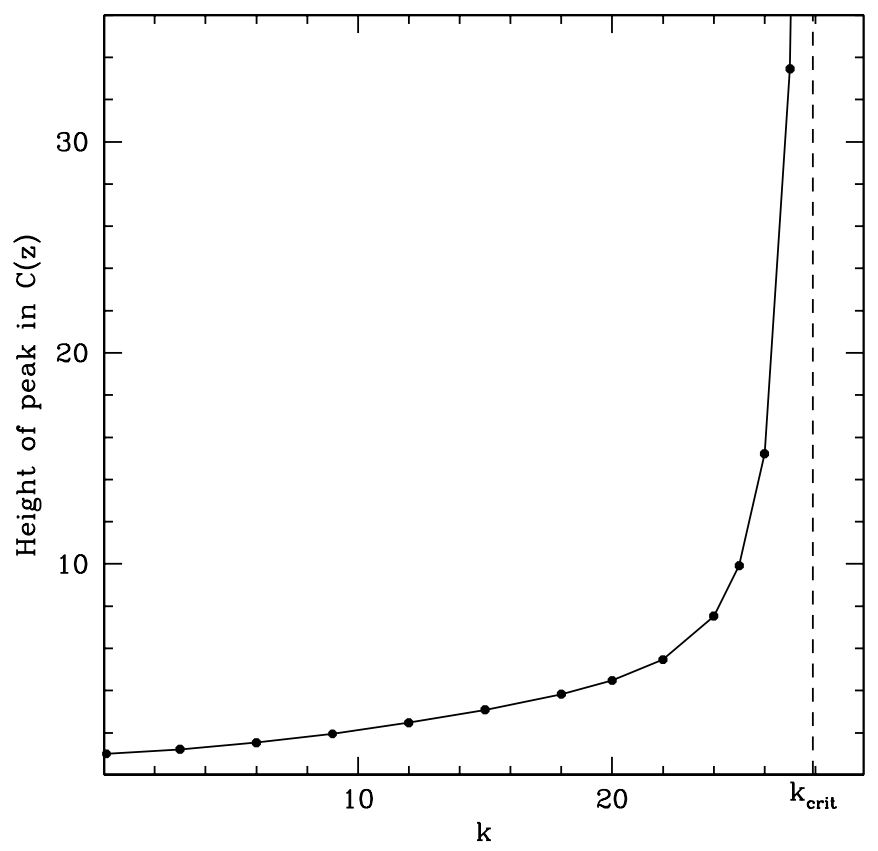

FIG. 6. Illustration of the variation with $k$ of the height of the peak in the pulse wave $C(z)$. This height $\rightarrow \infty$ as $k \rightarrow k_{\text {crit. }}^{-}$. The values illustrated are for $h($.$) as in Figure 5$, for which $k_{\text {crit }} \approx 28$. For $k>k_{\text {crit }}$ there are no traveling wave solutions.

The function $\widetilde{\gamma}($.$) has exactly the same qualitative form as \gamma($.$) and moreover$ satisfies the key inequality $\widetilde{\gamma}(W) \geq \widetilde{\gamma}(0)$ when $W<0$. Therefore, the proof in section 3 of the existence of a heteroclinic connection between $(1,0)$ and $(0,0)$ in the $U-W$ phase plane holds for this amended system, as does the proof of (i)-(iii) in section 4. Moreover, since $\widetilde{\gamma}(0)$ is independent of $\theta($.$) , the minimum wave speed is not affected$ by the change in the model. Therefore, for wave speeds greater than or equal to this minimum value, a traveling wave solution of (9) exists for all values of $k$ when $h($.$) and$ $\theta($.$) are such that C \rightarrow \infty$ as $U^{\prime} \rightarrow-\infty$, that is, when $h(\infty) \cdot \theta(\infty)=0$. Conversely, when both $h(\infty)$ and $\theta(\infty)$ are nonzero, a traveling wave solution exists for $k$ below a unique, critical value.

Intuitively, one expects that for given functions $h($.$) and f($.$) , and a given value$ of $k$ for which traveling waves exist for both (1) and (9), the peak in the $C$ wave form will be higher for (1) than for (9); recall that the change made to the model in this section corresponds to limiting the extent of matrix convection. In the remainder of this section, I will show that this intuitive expectation is indeed correct. The natural approach to use for this proof would be to compare the two ordinary differential equation systems (7) and (13). However, I have been unable to make any progress using this approach because of the difficulty of comparing $\gamma($.$) and \widetilde{\gamma}($.$) . Since \theta^{\prime}(C) \leq 0$ for all $C$, comparison of (6) and (12) shows immediately that for a given value of $C>1$, $\left[1 / \Phi^{\prime}(C)\right]>\left[1 / \widetilde{\Phi}^{\prime}(C)\right]$. However, the corresponding inequality cannot be extended to $\gamma(W)$ and $\widetilde{\gamma}(W)$, since these are not necessarily monotonic functions, and this makes comparison of trajectories difficult in the $U-W$ plane.

Therefore I adopt the different approach of working in the $U-C$ phase plane. This is a more difficult system to work with when considering the existence of traveling 
waves but makes comparison of wave forms easier. The equations follow directly from (3) and (10):

$$
\begin{aligned}
& U^{\prime}=\frac{-a(1-1 / C)}{k h(C)}, \\
& C^{\prime}=\left[k C^{2} f(U) / a-a C(C-1) / h(C)\right]
\end{aligned}
$$

for the original model (1), and

$$
\begin{aligned}
& U^{\prime}=\frac{-a(1-1 / C)}{k \theta(C) h(C)} \\
& C^{\prime}=\left[k \theta(C) C^{2} f(U) / a-a C(C-1) / h(C)\right] /\left[1+C(1-C) \theta^{\prime}(C) / \theta(C)\right]
\end{aligned}
$$

for the amended model (9). In this system of ordinary differential equations, a traveling wave solution corresponds to a heteroclinic connection between the equilibrium points $(0,1)$ and $(1,1)$. I am concerned with the comparison of these solutions for given $h(),. f(),$.$a , and k$ such that both systems have a traveling wave solution. I denote the corresponding trajectories in the $U-C$ plane by $\mathcal{T}$ for (1) and $\widetilde{\mathcal{T}}$ for (9), and following my previous notation, I denote the points on these trajectories at which $C$ has its (unique) local maximum as $U_{m i n}$ and $\widetilde{U}_{m i n}$, respectively.

I now consider the portion $U \in\left(\widetilde{U}_{m i n}, 1\right)$ of the trajectory $\widetilde{\mathcal{T}}$. Using (15), it follows that along this curve,

$$
\begin{aligned}
& \frac{d C}{d U}<0 \Rightarrow k \theta(C) C f(U) / a>a(C-1) / h(C) \\
& (16) \quad \Rightarrow[a(C-1) / h(C)-k C f(U) / a]<[a(C-1) / h(C)-k \theta(C) C f(U) / a]<0 .
\end{aligned}
$$

This inequality can be used when considering the value of $d C / d U$ in the phase plane of the original model, (14), for which

$$
\frac{d C}{d U}=\frac{k C^{2} h(C)}{a(C-1)}\left[\frac{a(C-1)}{h(C)}-k C f(U) / a\right] .
$$

Therefore along the portion $U \in\left(\widetilde{U}_{\text {min }}, 1\right)$ of the curve $\widetilde{\mathcal{T}}$ (which is of course not a trajectory for these equations),

$$
\begin{aligned}
\frac{d C}{d U} & <\frac{k C^{2} h(C)}{a(C-1)}\left[\frac{a(C-1)}{h(C)}-k C \theta(C) f(U) / a\right] \quad \text { using (16) } \\
& \leq \frac{\theta(C) k C^{2} h(C)}{a(C-1)}\left[\frac{a(C-1)}{h(C)}-k C \theta(C) f(U) / a\right] /\left[1+C(1-C) \theta^{\prime}(C) / \theta(C)\right],
\end{aligned}
$$

which is the slope of $\widetilde{\mathcal{T}}$. Here I am using the conditions $\theta(C) \leq 1, C>1$, and $\theta^{\prime}(C) \leq 0$.

Therefore, in the system (14), all trajectories pass through the portion $U \in$ $\left(\widetilde{U}_{\text {min }}, 1\right)$ of the curve $\widetilde{\mathcal{T}}$ in the direction of increasing $C$. Moreover, $U^{\prime}<0$ along the line $U=\widetilde{U}_{\text {min }}, C>1$, while along $C=1, U \in(0,1)$, (14b) implies that $C^{\prime}=k f(U) / a>0$. Thus, all trajectories leave the set $\mathcal{S}$, defined as the portion of the phase plane between $\widetilde{\mathcal{T}}, C=1$, and $U=\widetilde{U}_{\text {min }}$. The slopes of $\mathcal{T}$ and $\widetilde{\mathcal{T}}$ are actually the same at $(1,0)$ (the eigenvector is independent of $\theta($.$) ), but expansion to$ quadratic terms near $(1,0)$ shows that $\mathcal{T}$ lies outside $\mathcal{S}$ for $U$ close to 1 . Hence, $\mathcal{T}$ must 


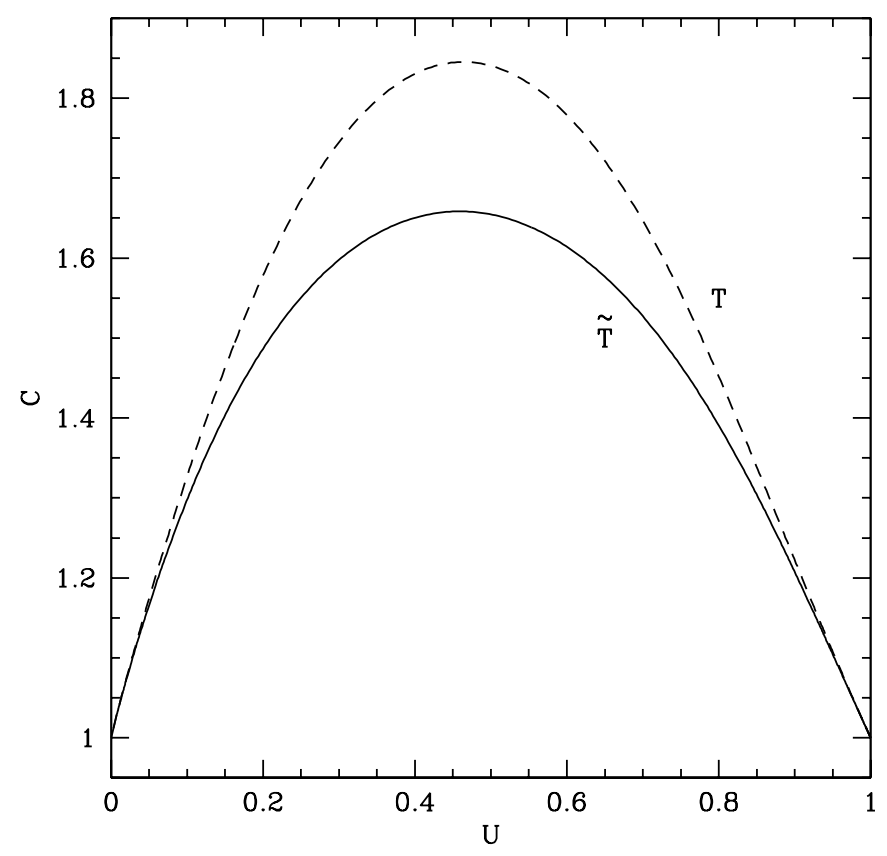

FIG. 7. A comparison between the traveling wave trajectories, plotted in the $U-C$ plane, with (solid line, labeled $\widetilde{\mathcal{T}}$ ) and without (dashed line, labeled $\mathcal{T}$ ) the inclusion of a function $\theta(C)$ to represent saturation in the matrix rearrangement potential of tumor cells. The height of the $C$ wave is reduced by the inclusion of this saturation effect. The trajectories were determined by numerical integration of the two sets of traveling wave ODEs with $h(C)=[1-\tanh (c / 2-1)] / 2$ and $\theta(C)=$ $[1-\tanh (c / 2-1)] /[1+\tanh (1 / 2)]$.

remain outside $\mathcal{S}$ for $U \in\left(\widetilde{U}_{\text {min }}, 1\right)$ and thus must reach a value of $C$ that is at least as large as the value of $C$ on $\widetilde{\mathcal{T}}$ at $U=\widetilde{U}_{\text {min }}$. Thus the traveling wave in the original model, corresponding to $\mathcal{T}$, has a maximum value of $C$ at least as large as that for the amended model (that is, the wave corresponding to $\widetilde{\mathcal{T}}$ ). Figure 7 illustrates a typical comparison between the two trajectories.

7. Discussion. The mechanisms by which many solid tumors acquire a dense, fibrous capsule remain unknown, despite it being a very prominent morphological feature with major clinical significance. The model studied in this paper investigates the "expansive growth hypothesis," that is, the possibility that the capsule forms by remodeling of existing extracellular matrix, without any new matrix production. The model solutions all have the form of a pulse of extracellular matrix moving ahead of the growing tumor, corresponding to a fibrous capsule. I have shown that the existence of such a solution is a robust consequence of the expansive growth hypothesis, applying when the basic model of [14] is extended to include decreases in the cell speed and/or matrix reorganization capacity per cell, irrespective of the form of these saturation effects.

Moreover, I have shown that the predicted density of matrix in the capsule correlates directly with the parameter $k$, which represents the rate of local matrix movement and remodeling per cell. Very significantly, this implies that the capsule density is not correlated with either the speed at which the tumor grows (which is independent of $k$ ) or its size. This explains the wide discrepancies between studies attempting to 
correlate tumor size and capsule incidence or thickness [12], [17], [13], [6] and argues against the conventional intuition that these should be correlated if the capsule forms without matrix production. The key experimental test of the model would be to study the correlation between capsule density and some measure of the parameter $k$. This is not possible using pathology data alone but may be possible for animal studies of the encapsulation of tumor implants, of the type performed by Vaage and Harlos [16]. This would involve culturing cells from the implant in vitro and comparing the $k$ value of the cultured cells.

Mathematically, the proof of the existence of traveling wave solutions in a system of equations is unusual and is made possible by the absence of a kinetic term in (1b), so that the corresponding traveling wave equation can be integrated once exactly. This avoids the need to work in a phase space of more than two dimensions, where proof of existence of a heteroclinic connection is notoriously difficult [4]. Generalization of the analysis to the case of new matrix secretion occurring, which would enable investigation of the expansive growth hypothesis in a broader context, thus remains a significant challenge for future work.

Acknowledgments. This work was made possible by inspiring discussions with Abbey Perumpanani, during our collaboration on previous work, and I am very grateful to him. I also thank John Dallon, Dugald Duncan, Chris Eilbeck, and Pam Wiener for helpful discussions.

\section{REFERENCES}

[1] M.R. Booty, R. Haberman, And A.A. Minzoni, The accommodation of traveling waves of Fisher's type to the dynamics of the leading tail, SIAM J. Appl. Math., 53 (1993), pp. 1009-1025.

[2] D. Bray, Cell Movements, Garland, New York, 1992.

[3] P.N. Brown, G.D. Byrne, and A.C. Hindmarsh, VODE: A variable-coefficient ODE solver, SIAM J. Sci. Statist. Comput., 10 (1989), pp. 1038-1051.

[4] S.R. Dunbar, Traveling wave solutions of diffusive Lotka-Volterra equations, a heteroclinic connection in $R^{4}$, Trans. Amer. Math. Soc., 286 (1984), pp. 557-594.

[5] H.L. Evans, Encapsulated columnar cell neoplasms of the thyroid-a report of four cases suggesting a favorable prognosis, Amer. J. Surg. Pathol., 20 (1996), pp. 1205-1211.

[6] K. Kishi, T. Shikata, S. Hirohashi, H. Hasegawa, S. Yamazaki, and M. Makuuchi, Hepatocellular carcinoma, a clinical and pathological analysis of 57 hepatectomy cases, Cancer, 51 (1983), pp. 542-548.

[7] A. Kolmogoroff, I. Petrovskit, and N. Piscounoff, Etude de l'équation de la diffusion avec croissance de la quantité de matiére et son application á un problème biologique, Moscow Bull. Math., 1 (1937), pp. 1-25.

[8] E.C.S. Lai, I.O.L. NG, M.M.T. NG, A.S.F. LoK, P.C. Tam, S.T. Fan, T.K. Choi, and J. WONG, Long-term results of resection for large hepatocellular carcinoma, a study of 26 cases, Hepatology, 11 (1990), pp. 815-818.

[9] D.A. Larson, Transient bounds and time-asymptotic behavior of solutions to nonlinear equations of Fisher type, SIAM J. Appl. Math., 34 (1978), pp. 93-103.

[10] A. Moreno, J.M. Rodriguez, J. Sola, T. Soria, and P. Parrilla, Encapsulated papillary neoplasm of the thyroid - retrospective clinicopathological study with long-term follow-up, European J. Surg., 162 (1996), pp. 177-180.

[11] W. Morton and D. Mayers, Numerical Solution of Partial Differential Equations, Cambridge University Press, Cambridge, UK, 1994.

[12] I.O.L. NG, E.C.S. LAI, M.T. NG, AND S.T. FAN, Tumor encapsulation in hepatocellular carcinoma, Cancer, 70 (1992), pp. 45-49.

[13] K. Okuda, R.L. Peters, AND I.W. Simson, Gross anatomic features of hepatocellular carcinoma from three disparate geographic areas, proposal of new classification, Cancer, 54 (1984), pp. 2165-2173. 
[14] A.J. Perumpanani, J.A. Sherratt, and J. Norbury, Mathematical modelling of capsule formation and multinodularity in benign tumor growth, Nonlinearity, 10 (1997), pp. 15991614.

[15] J.A. Sherrate, On the transition from initial data to traveling waves in the Fisher-KPP equation, Dynam. Stability Systems, 13 (1998), pp. 167-174.

[16] J. VAaGe AND J.P. HARLos, Collagen production by macrophages in tumor encapsulation and dormancy, British J. Cancer, 63 (1991), pp. 758-762.

[17] K. Wakasa, M. Sakurai, J. Okamura, and C. Kuroda, Pathological study of small hepatocellular carcinoma, frequency of their invasion, Virchows Arch. A, 407 (1985), pp. 259-270. 